\title{
Safety and immunogenicity of intradermal rabies vaccination for post exposure prophylaxis
}

\author{
Ravish S. Hardanahalli ${ }^{1}$, Rachana R. Annadani ${ }^{2}$, Madhusudana N. Shampur ${ }^{3}$, \\ Veena Vijayashankar ${ }^{4}$, Ashwath Narayana H. Doddabele ${ }^{1}$, Gangaboraiah ${ }^{1}$, Malatesh Undi ${ }^{2}$
}

\begin{abstract}
${ }^{1}$ Department of Community Medicine, Kempegowda Institute of Medical Sciences, Banashankari II stage, Bangalore 70, Karnataka, India

${ }^{2}$ Department of Community Medicine, SS Institute of Medical Sciences \& Research Centre, Davangere, Karnataka, India

${ }^{3}$ Department of Neurovirology, National Institute of Mental health and Neurosciences (NIMHANS), Hosur Road, Bangalore 560029, Karnataka, India

${ }^{4}$ Department of Community Medicine, BGS Global Institute of Medical Sciences, Uttarahalli, Kengeri, Bangalore, Karnataka, India
\end{abstract}

Received: 11 January 2016

Accepted: 08 February 2016

\author{
*Correspondence: \\ Dr. Ravish S. Hardanahalli, \\ E-mail: drravishhs@gmail.com
}

Copyright: () the author(s), publisher and licensee Medip Academy. This is an open-access article distributed under the terms of the Creative Commons Attribution Non-Commercial License, which permits unrestricted non-commercial use, distribution, and reproduction in any medium, provided the original work is properly cited.

\section{ABSTRACT}

Background: The affordability to anti-rabies vaccine (ARV) for intramuscular administration in post exposure prophylaxis (PEP) is a major constraint. Therefore, in countries, where there are financial constraints, WHO recommends intradermal rabies vaccination (IDRV) that reduces the quantity and cost of vaccination. The aim of the study was to evaluate the safety and immunogenicity of IDRV implemented under National pilot project.

Methods: A longitudinal study was conducted at anti rabies clinic (Government referral hospital), India where IDRV is implemented. The study included 515 animal bite cases who received PEP as recommended by WHO. ARV was administered intradermally using updated Thai red-cross regimen.

Results: The incidence of adverse drug events was $9.7 \%$ and all resolved without any complication. The geometric mean concentration of rabies virus neutralizing antibodies among the vaccinees was $11.89 \mathrm{IU} / \mathrm{mL}$ on day 14 , which was above the WHO recommended titers of $\geq 0.5 \mathrm{IU} / \mathrm{mL}$.

Conclusions: IDRV was found to be safe and immunogenic in PEP.

Keywords: Animal bites, Intradermal rabies vaccination, Safety, Immunogenicity

\section{INTRODUCTION}

Rabies is a fatal encephalitis that occurs in $>100$ countries throughout the world. It is transmitted to humans and other animals through close contact with saliva from infected animals i.e. bite, scratches, licks on broken skin and mucous membranes. Although a number of animals serve as vectors for transmission, dogs are the main source of human infections and poses a potential threat to $>3.3$ billion people worldwide. ${ }^{1}$ Timely and correct post exposure prophylaxis (PEP) for these animal bite victims is necessary to prevent rabies. Proper wound management and simultaneous administration of rabies immunoglobulins (RIG) in all category III exposures combined with prompt administration of potent cell culture vaccines $(\mathrm{CCV})$ is effective in preventing rabies, even after high-risk exposure. ${ }^{2}$

Since their development, more than four decades ago, CCVs have proved to be safe and effective in preventing 
rabies. These vaccines are intended for both pre- and post-exposure prophylaxis and have been administered to millions of people worldwide. ${ }^{3}$ But, the affordability to CCVs for intramuscular administration during PEP is a major constraint in developing countries of Asia and Africa. Therefore, World Health Organization (WHO) recommends intradermal route of vaccination with $\mathrm{CCVs}$ for these countries to reduce the quantity of vaccine and the cost of vaccination. ${ }^{2}$

In India, animal bites in humans are a major public health problem and an estimated 17.4 million animal bites occur annually which accounts to an incidence of $1.7 \%{ }^{4}$ Considering the large number of animal bite cases in the country and huge demand for CCVs, there is a need to introduce intradermal rabies vaccination (IDRV). Therefore, following the recommendations of WHO and ICMR, the drug controller general of India (DCGI) approved intra dermal administration of rabies vaccines using updated TRC regimen in 2006.

Department of health and family welfare, Government of India, under $11^{\text {th }}$ five year plan, implemented pilot project on prevention and control of rabies in humans in 5 cities through national centre for disease control (NCDC) as the nodal agency. The main strategy is to make IDRV operational and to facilitate wider coverage of PEP with the available quantity of vaccines and make PEP cost effective. ${ }^{5}$ Bangalore is one of the project areas where it is implemented \& Banashankari corporation referral hospital is one of the six centers in Bangalore, where pilot project is implemented. The present study was done to evaluate the safety and immunogenicity of intradermal rabies vaccination among animal bite victims in this hospital.

\section{METHODS}

This longitudinal study was conducted for a period of one and half years at anti rabies clinic, Banashankari corporation referral hospital, Bangalore, India where IDRV is implemented. 515 animal bite cases, who reported to the study centre for post exposure prophylaxis during the study period, were included in the study based on the inclusion and exclusion criteria. A thorough and detailed enquiry was done among all the study subjects to rule out taking any rabies vaccine either as pre exposure prophylaxis (PrEP) or PEP and history of any animal bite in the past. Similarly, any concomitant medical conditions / treatments were ruled out.

A standard case record form was maintained for each bite victim that included details of socio-demographic profile, type of exposure and post exposure prophylaxis provided at the study centre and the telephone number for further follow up.

The study was initiated, following clearance from the institutional ethics committee and was conducted in accordance with ICH - GCP guidelines. All the bite victims were given post exposure prophylaxis (PEP) including wound wash, anti-rabies vaccine and equine rabies immunoglobulin (ERIG) in all category III bites. Anti rabies vaccine was administered intradermally using updated Thai red cross regimen i.e., 2 doses of $0.1 \mathrm{ml}$ vaccine given over both the deltoid muscle on days 0,3 , 7 and 28. The vaccines used during the period of study were Rabipur (Purified chick embryo vaccine, manufactured by Novartis vaccines) and Abhayrab (Purified vero cell rabies vaccine, manufactured by Human biological institute).

\section{Assessment of safety}

Following vaccination, all the subjects were observed for half an hour for possible immediate local/ systemic adverse drug events (ADEs). At the end of half an hour, reactogenicity was recorded, only if the subject spontaneously complained of a problem to a question on general wellbeing i.e., unaided recall. The subjects were given a follow up card to indicate if they had any late adverse events and was recorded in the subsequent visits i.e., on Day 3, 7 and 28.

\section{Assessment of immunogenicity}

Blood samples were drawn from a sub sample of 95 subjects who consented on Day 14 for estimation of rabies virus neutralizing antibody (RVNA). $5 \mathrm{ml}$ of venous blood was drawn from each patient under aseptic precautions and the sera were separated and tested for RVNA by rapid fluorescent focus inhibition test (RFFIT) at the Department of neurovirology, National Institute of Mental Health and Neurosciences (NIMHANS), Bangalore, India which is a WHO collaborating centre for reference and research on rabies.

\section{Estimation of rabies virus neutralizing antibody (RVNA)}

Modified RFFIT was done as per WHO recommended procedure. The cell line used was BHK 21 (ATCC CCL 10) and 96 well tissue culture plates (Sigma) and BHK21 adapted CVS 11 strain of rabies virus. The reference serum used was an in house serum calibrated against $2^{\text {nd }}$ international reference standard having a titer of 30 $\mathrm{IU} / \mathrm{mL}$ (obtained from National Institute of Biological standards, UK). Briefly, doubling dilutions of serum samples and reference serum (after heat inactivation at 56 $\mathrm{C}$ for $30 \mathrm{~min}$ in a water bath) in duplicate were made in 96 well plates using IMDM (Sigma Cat No.17633) To each $100 \mu \mathrm{l}$ of serum dilution $100 \mu \mathrm{l}$ of CVS (100 FFD ${ }_{50}$ ) was added and the plate to was incubated at $37 \mathrm{C}$ for one hour. A confluent monolayer of BHK 21 cells were trypsinized and re- suspended in $10 \mathrm{ml}$ of IMDM with 10\% FCS (Sigma, cat No. F2442). Cell control and virus controls were also included. To each well of the 96 well plates $100 \mu \mathrm{l}$ of cell suspension was added and the plate was incubated at $37 \mathrm{C}$ in a $\mathrm{CO} 2$ incubator (Sanyo, Japan). After 24 hours the cells were fixed in cold acetone for 30 minutes and stained by direct FAT using commercially 
available rabies $\mathrm{N}$ conjugate (light diagnostics USA, Cat No. F199). The plates were then observed under an inverted fluorescence microscope (Nikon Eclipse). The highest dilution of serum showing $50 \%$ inhibition of fluorescence foci was taken as end point dilution. The titer was converted to $\mathrm{IU} / \mathrm{mL}$ in comparison with reference serum.

All the study subjects were followed up for 1 year to know their survival status.

All the biting dogs could not be traced or caught for laboratory examination due to logistical difficulties.

\section{Statistical analysis}

The data was analysed statistically by computing percentages, geometric mean concentration (GMC), range, geometric standard deviation (GSD), Standard error (SE), 95\% confidence interval (CI) for GMC.

\section{RESULTS}

All the belonged to lower or lower 515 animal bite victims were included in the study, majority of them were in the age group of $16-45$ years $(46.21 \%)$ followed by 7 15 years $(27.77 \%)$ with the median age being 17.48 years and inter quartile range of $11-37$ years. $351(68.16 \%)$ were males and $164(31.84 \%)$ were females. Most of the study subjects i.e., $215(41.75 \%)$ were illiterates and belonging to lower socio-economic status (62.91\%) (Table 1).

Table 1: Socio demographic characteristics of animal bite victims.

\begin{tabular}{|lll|l|}
\hline Characteristics & Number & Percentage \\
\hline \multirow{4}{*}{ Age } & $0-6$ & 54 & 10.48 \\
& $7-15$ & 143 & 27.77 \\
& $16-45$ & 238 & 46.21 \\
& $46-60$ & 52 & 10.10 \\
\multirow{2}{*}{ Sex } & $>60$ & 28 & 5.44 \\
\hline \multirow{4}{*}{ Male } & Female & 351 & 68.16 \\
& Illiterate & 164 & 31.84 \\
\hline \multirow{4}{*}{ Education } & Primary school & 215 & 41.75 \\
& Middle school & 83 & 16.12 \\
& High school & 44 & 8.54 \\
& Intermediate & 83 & 16.12 \\
& Graduate/Postgrad & 48 & 9.32 \\
& uate Professional & 37 & 7.18 \\
& degree & 5 & 0.97 \\
Socio- & Upper & 12 & 2.33 \\
economic & Upper middle & 28 & 5.44 \\
status* & Lower middle & 142 & 27.57 \\
& Upper lower & 324 & 62.91 \\
& Lower & 9 & 1.75 \\
\hline
\end{tabular}

*Modified Kuppuswamy Socioeconomic Status classification
In the present study, the biting animals were dog $(97.28 \%)$, cat $(1.55 \%)$ and monkey $(1.17 \%)$. The site of bite was on lower limb (68.93\%), upper limb (21.17\%), trunk $(6.60 \%)$, head and neck $(2.72 \%)$ and multiple sites $(0.58 \%)$. Majority $(75.53 \%)$ of the animal bite victims had category III exposure. $67 \%$ of the study subjects had done wound wash with soap and water. None of the biting animal was followed due to logistical reasons (Table 2).

Table 2: Distribution of study subjects according to the details of exposure.

\begin{tabular}{|c|c|c|c|}
\hline Characteristics & & $\begin{array}{l}\text { Number } \\
(n=515)\end{array}$ & Percentage \\
\hline \multirow{3}{*}{ Biting animal } & Dog & 501 & 97.28 \\
\hline & Cat & 8 & 1.55 \\
\hline & Monkey & 6 & 1.77 \\
\hline \multirow{2}{*}{ Type of dog } & Pet dog & 177 & 34.37 \\
\hline & Stray dog & 324 & 62.91 \\
\hline \multirow{2}{*}{$\begin{array}{l}\text { Category of } \\
\text { exposure }\end{array}$} & III & 389 & 75.53 \\
\hline & II & 126 & 24.47 \\
\hline \multirow{5}{*}{ Site of bite } & Head \& neck & 14 & 2.72 \\
\hline & Trunk & 34 & 6.60 \\
\hline & Upper limb & 109 & 21.17 \\
\hline & Lower limb & 355 & 68.93 \\
\hline & Multiple site & 3 & 0.58 \\
\hline \multirow{4}{*}{$\begin{array}{l}\text { Type of } \\
\text { wounds }\end{array}$} & Abrasion & 251 & 48.74 \\
\hline & Laceration & 56 & 10.87 \\
\hline & $\begin{array}{l}\text { Puncture } \\
\text { wounds }\end{array}$ & 176 & 34.17 \\
\hline & Mixed wounds & is 32 & 6.21 \\
\hline \multirow{2}{*}{$\begin{array}{l}\text { Wound wash } \\
\text { following bite }\end{array}$} & Yes & 345 & 67 \\
\hline & No & 170 & 33 \\
\hline \multirow{2}{*}{$\begin{array}{l}\text { Application of } \\
\text { local irritant }\end{array}$} & Yes & 79 & 15.34 \\
\hline & No & 436 & 84.66 \\
\hline
\end{tabular}

The incidence of adverse drug events (ADEs) was found to be $9.7 \%$. The ADEs were pain at the site of injection, induration, erythema, itching \& fever. All the ADEs were mild and resolved without any complications (Table 3 ).

The RVNA response among all the subjects on day 14 was adequate as per WHO recommendation of $\geq 0.5$ $\mathrm{IU} / \mathrm{ml}$ with the geometric mean concentration (GMC) of $11.89 \mathrm{IU} / \mathrm{mL}$. All the study subjects were healthy and alive after one year of completing PEP (Table 4).

Table 3: Adverse drug events among the study subjects.

\begin{tabular}{|ll|}
\hline Adverse drug events & Number $(\%)$ \\
\hline Erythema & $11(2.1 \%)$ \\
\hline Itching & $16(3.1 \%)$ \\
\hline Pain & $11(2.1 \%)$ \\
\hline Induration & $4(0.8 \%)$ \\
\hline Fever & $8(1.6 \%)$ \\
\hline Total & $50 / 515(9.7 \%)$ \\
\hline
\end{tabular}


Table 4: Immunogenicity among the study subjects.

\begin{tabular}{|llllll|}
\hline No. of subjects & GMC (IU/mL) (day 14) & GSD (IU/mL) & SE (GMC) & $\begin{array}{l}\text { 95\% CI } \\
\text { Lower bound }\end{array}$ & Upper bound \\
\hline 95 & 11.89 & 1.32 & 0.20 & 11.50 & 12.28 \\
\hline
\end{tabular}

GMC- Geometric Mean Concentration; GSD- Geometric Standard Deviation; SE-Standard Error, CI- Confidence Interval

\section{DISCUSSION}

India is highly endemic for rabies and has the largest number of animal bites in the world. Cell culture vaccines given intramuscularly are the main stay of PEP for animal bite victims. Intradermal administration of cell culture rabies vaccines offers an equally safe and immunogenic alternative to intramuscular rabies vaccination and requires less volume of vaccine and is recommended by WHO in resource constraint countries. Thus, intradermal rabies vaccination reduces the direct cost of vaccine by $60-80 \%$ compared with standard intramuscular vaccination. ${ }^{1}$

The present study showed that, intradermal rabies vaccination is safe for post exposure prophylaxis. Similar results were shown in a study conducted by Madhusudana et al with ADEs of $9.5 \%$ with the use of purified cell culture rabies vaccine (PCECV), and study by Sudarshan, et al with PCECV using KIMS regimen also showed a total ADEs of 3.1. ${ }^{6,7}$ Similarly, Sampath et al also showed that, the total ADEs of $5.50 \%$ for purified vero cell rabies vaccine. ${ }^{8}$ Therefore, the safety of study vaccines was comparable to other studies and it confirms that IDRV is safe for PEP among animal bite victims.

World Health Organization recommends a minimum RVNA titer of $\geq 0.5 \mathrm{IU} / \mathrm{mL}$ of serum for protection against rabies from day 14 onwards. ${ }^{1}$ The objective of vaccination in post-exposure prophylaxis (PEP) is to stimulate the immune system to produce antibody titers of at least $0.5 \mathrm{IU} / \mathrm{mL}$ by day 14 and persist for a long time in animal bite cases. In the present study, all animal bite victims had protective RVNA titers of $\geq 0.5 \mathrm{IU} / \mathrm{mL}$ on day 14 with the Geometric Mean Concentration of 11.89. Similarly, a study conducted by Khawplod et al showed GMC titers of $9.14 \mathrm{IU} / \mathrm{mL}$ on day $14 .^{9}$ Another study conducted by $\mathrm{P}$ Suntharasamai et al, showed the GMC of $9.07 \mathrm{IU} / \mathrm{mL}$ on day 14 , showing protective levels. ${ }^{10}$ Similarly, a study conducted by Madhusudana et al using TRC regimen showed GMC of $4.3 \mathrm{IU} / \mathrm{mL}$ on day $14 .^{6}$ In a study by Sudarshan, et al using KIMS regimen (2-2-2-2-2) also showed GMC of $4.17 \mathrm{IU} / \mathrm{mL}$ on day 14 . $^{7}$ The immunogenicity of the intradermal rabies vaccines in the present study were similar to other studies and it confirms the immunogenicity of IDRV among animal bite victims.

\section{CONCLUSION}

In conclusion, the present study showed that, the intradermal rabies vaccination using Updated TRC regimen (2-2-2-0-2) was safe and immunogenic as PEP in animal bite victims. Therefore, IDRV will have an important role in effective post exposure prophylaxis and thereby eliminating rabies in India and other Asian countries.

\section{Funding: No funding sources} Conflict of interest: None declared

Ethical approval: The study was approved by the Institutional Ethics Committee

\section{REFERENCES}

1. Weekly Epidemiological Record. World Health Organization (WHO) Rabies vaccines, WHO position paper. 2007;49/ 50(82):425-35.

2. WHO Expert Consultation on Rabies: Second report, Technical Report Series 982. WHO Geneva. 2013:54-5.

3. WHO Expert Consultation on Rabies: First Report, Technical Report Series 931. WHO Geneva. 2005;26-7.

4. Sudarshan MK, Mahendra BJ, Madhusudana SN, Ashwath Narayana DH, Rahaman A, Rao NSN. An epidemiological study of animal bites in India: results of a WHO sponsored national multi-centric rabies survey. J Commun Dis. 2006;38(1):32-9.

5. National guidelines for rabies prophylaxis and intradermal administration of cell culture rabies vaccines: National Institute of Communicable Diseases, Ministry of Health \& Family welfare, New Delhi, India. 2007;14-5.

6. Madhusudana SN, Sanjay TV, Mahendra BJ, Suja MS: Simulated post-exposure rabies vaccination with purified chick embryo cell vaccine using a modified Thai Red Cross regimen. Int J Infect Dis. 2004;8(3):175-9.

7. Sudarshan MK, Madhusudana SN, Mahendra BJ, Ashwathnarayana DH, Anand Giri MS, Popova O. Evaluation of a new five injection, two site, intradermal schedule for purified chick embryo cell rabies vaccine: A randomized, open label active controlled trial in healthy adult volunteers in India. Current therapeutic research. 2005;66(4):323-4.

8. Sampath G, Suhasini VR, Lakshmiprasad RM, Udayabhaskara RY, Palaniappan C: An immunogenicity study of a newly introduced vero cell rabies vaccine (Abhayrab) manufactured in India. Vaccine. 2005;23(7):897-900.

9. Khawplod P, Tantawichien T, Wilde H, Limusanno S, Tantawichien T, Saikasem A. Use of Rabies 
vaccines after Reconstitution and Storage. Clin Infect Dis. 2002;34:404-6.

10. Suntharasamai P, Chaiprasithikul P, Wasi C, Supanaranond W, Auewarakul P, Chanthavanich P: A simplified and economical intradermal regimen of purified chick embryo cell rabies vaccine for post exposure prophylaxis. Vaccine. 1994;12(6):508-12.
Cite this article as: Hardanahalli RS, Annadani RR, Shampur MN, Vijayashankar V, Doddabele ANH, Gangaboraiah, et al. Safety and immunogenicity of intradermal rabies vaccination for post exposure prophylaxis. Int J Community Med Public Health 2016;3:692-6. 